Han et al, Afr J Tradit Complement Altern Med., (2017) 14 (4): 239-246

https://doi.org/10.21010/ajtcam.v14i4.26

\title{
MECHANISMS OF COIX SEED COMPOSITIONS IN THE TREATMENT OF SPLEEN DEFICIENCY AND WET DAMPNESS ZHENG
}

\author{
Xiaochun Han ${ }^{[1] *}$, Xuming $\mathrm{Ji}^{[1] *}$, Haijun Zhao ${ }^{[1]}$, Yanan Zhang ${ }^{[1]}$, Guowei Liu ${ }^{[2]}$, Yanfang Wang ${ }^{[1]}$, \\ Wenxiao Zhao ${ }^{[3]}$, Shijun Wang ${ }^{[1]^{*}}$
}

${ }^{[1]}$ College of Traditional Chinese Medicine, Shandong University of Traditional Chinese Medicine, Jinan, China. ${ }^{[2]}$ College of Foreign Languages, Shandong University of Traditional Chinese Medicine, Jinan, China. ${ }^{[3]}$ College of Nursing, Shandong University of Traditional Chinese Medicine, Jinan, China. ${ }^{*}$ Same contributions

*Corresponding Author Email: pathology@163.com

\begin{abstract}
Background: Coix seed has the functions of fortifying the spleen and inhibiting the dampness. However, it remains unclear which Coix seed compositions is responsible for these functions. Previous investigations have revealed that the main compositions of Coix seed are proteins, polysaccharides, oils and starches. The objectives of this study are to explore which is the most effective compositions in fortifying the spleen and examine how Coix seed works in regulating the water transport on the spleen deficiency and wet dampness (SDWD) rat model. Materials and Methods: The rats used were divided into (i) control group, (ii) model group, (iii) decoction group, (iv) protein group, (v) polysaccharide group, (vi) oil group and (vii) starch group. The urine volume, the drinking volume and the water loading index in each group were calculated. Agilent $8 * 60 \mathrm{~K}$ array was used for microarray-based gene expression analysis. The differential mRNAs related to the transport activity were screened. qRT-PCR was used to validate the mRNA microarray.

Results: The results demonstrated that all treatment groups could decrease the dampness of SDWD rats. mRNA microarray had significant effect on the protein group and the polysaccharide group in regulating the water transport, among which the most significant mRNA was Fabp6, Slc51a, Slc51b, Slc11a2, Slc4a10 and AQP3 respectively.

Conclusion: The compositions of proteins and polysaccharides had the most significant effect in regulating the water transport of SDWD rat model. The contributing mRNA focused on Fabp, Slc and AQP family.
\end{abstract}

Key words: spleen deficiency and wet dampness (SDWD), TCM, water transport, Coix seed, animal model.

\section{Introduction}

Spleen deficiency and wet dampness ZHENG (SDWD) is one of the major syndromes of traditional Chinese medicine (TCM) (Han et al., 2015). It is due to the improper diet, over fatigue, dysfunction of spleen and stomach and disorder of water transport. Clinically SDWD ZHENG is frequently treated with fortifying the spleen and inhibiting the dampness methods (Yang et al., 2016), among which Coix seed is one of the commonly-used drug (You-Ming et al., 2011).

Microarray-based gene expression analysis can be used to diagnose ZHENG, it also helps to explain the molecular mechanism of ZHENG and evaluate the drug effect in terms of changes of mRNA level (Liu et al., 2014). It has been applied to explain the nature of ZHENG and provide certain scientific evidence for ZHENG in TCM(Liu et al., 2016).

This study establishes SDWD rat model by high fat diet and exhaustive swimming. The model has been approved by National Patent Office (No.ZL201410072183.8). The model rats demonstrate obvious symptoms such as slow action and gastrointestinal dysfunction, which are consistent with the clinical manifestations of SDWD ZHENG (Cui et al., 2016, Jie et al., 2015).

Coix seed (Coixlacroyma-jobi L. var. ma-yuenStapf, adlay), used both as both as food and drug in TCM, has the function of fortifying the spleen and inhibiting the dampness. However, the effective component and the mechanism in fortifying the spleen and inhibiting the dampness remains obscure. In this study we establishes SDWD rat model to find the effective component of Coix seed and to clarify its mechanism. 


\section{Materials and Methods Animal model preparation}

70 Wistar rats (140-160 g, 35 male and 35 female) were obtained from Weitong Lihua Experiment Animals Inc. Ltd. (Beijing, China, No. SCXK 11400700067086). It took 8 weeks to establish 60 SDWD rat models by high fat diet and exhaustive swimming (GAO et al., 2014, Han et al., 2015). 10 rats in the control group had freedom of movement and received no intervention.

\section{Coix seed components}

The components such as proteins, polysaccharides, oils and starches were extracted individually from Coix seed, according to the methods in our previous reports (Cui et al., 2015; Cui et al., 2014; Wu et al., 2015).

\section{Administration mode and dosage}

All the model rats were divided into six groups randomly based on their weight and gender. $6.25 \mathrm{~g} \bullet \mathrm{kg}-1 \mathrm{~d}^{-1} \mathrm{Coix}$ seed was administrated to the decoction group based on the Chinese Pharmacopoeia (2010 Edition). $0.2813 \mathrm{~g} \bullet \mathrm{kg}-1 \mathrm{~d}^{-1}$ proteins component (equal to the proteins in the $6.25 \mathrm{~g} \cdot \mathrm{kg}-1 \mathrm{~d}^{-1}$ Coix seed) was administrated to the protein group. So did $0.9113 \mathrm{~g} \cdot \mathrm{kg}-1 \mathrm{~d}^{-1}$ polysaccharides component in the polysaccharide group, $0.2875 \mathrm{~g} \cdot \mathrm{kg}-1 \mathrm{~d}^{-1}$ oils component in the oil group, $1.7981 \mathrm{~g} \cdot \mathrm{kg}-1 \mathrm{~d}-1$ starches component in the starch group. And the model group was given normal saline. Drug was administered by gavage once a day for 14 days, $1 \mathrm{~mL} / 100 \mathrm{~g}$.

\section{Detection index}

After 2 weeks' treatment, the urine volume and the drinking volume were observed for 24 hours. Body mass was recorded $0 \mathrm{~h}, 1 \mathrm{~h}, 2 \mathrm{~h}, 4 \mathrm{~h}$, and $6 \mathrm{~h}$ after intraperitoneal injection with normal saline( $10 \%$ of body weight). Then the water loading index was calculated following the method described by Saito and Pedersen (Pedersen et al., 2003, Saito et al., 2001). The higher the water loading index was, the worse the water transport activity of the body was.

At the end of the experiment, 3 rats in each group were randomly selected for execution. Jejunum tissues from each rat were dissected and the total RNA was extracted for mixture in equal ratio. The mixed samples were subjected to microarray-based gene expression analysis

(Agilent Rat Gene Expression Version 6.5, 8*60K, Shanghai Quanmai Biological Technology Co., Ltd., Design ID: 028279). The criteria for screening differentially expressed mRNA was Fold Change>2 (FC $>2$ ). Geneontology (GO) was performed for differential mRNA according to the enrichment analysis by EMBL-EBI (http://www.ebi.ac.uk/services). The differential mRNAs related to transport activity in molecular function of GO terms were analyzed.

\section{Quantitative real-time PCR (qRT-PCR)}

qRT-PCR was used to validate the mRNA microarray. Total RNA was reverse-transcribed to cDNA with a reverse transcription kit (PrimerScript RT reagent Kit, Takara, USA). GAPDH was used as a reference. mRNA expression was detected by real-time PCR instrument (ABI, USA, $9700 \mathrm{HT}$ real-time system). ${ }^{\Delta \Delta} \mathrm{Ct}$ was calculated for relative quantification of mRNA expression.

\section{Statistical Analysis}

The urine volume, the drinking volume, and the water loading index were expressed as mean $\pm \mathrm{SD}$. One-way analysis of variance (ANOVA) and least significant difference (LSD- $t$ ) were used to compare the difference between groups. All the data were analyzed by IBM SPSS21.0 software. $P<0.05$ was accepted as statistical significance.

Differential mRNA related to transporter activity in molecular function was screened out from 7 groups. SIMCA-P 11.0 software was applied in the principal component analysis (PCA). The criteria for determining the number of principal components (PC) was cumulative contribution rate $>80 \%$.

\section{Results}

Covariance analysis of the urine volume in each group

The drinking volume affected the urine volume. After the influence of drinking was excluded, the urine volume in model group was lower than that of the control group $(P<0.01)$; the urine volume in the treatment groups was higher than that of the model group, especially that of the protein group $(P<0.05)$. (Table 1, Figure 1). 
Han et al, Afr J Tradit Complement Altern Med., (2017) 14 (4): 239-246

https://doi.org/10.21010/ajtcam.v14i4.26

Tabe1: Covariance analysis of urine volume in each group

\begin{tabular}{lrrrrrr}
\hline Source & Type III Sum of & Squares & df & Mean Square & F & Sig. \\
\hline Drinking & 21.943 & 1 & 21.943 & 4.683 & .034 \\
Urine & 130.285 & 6 & 21.714 & 4.634 & .001 \\
\hline
\end{tabular}

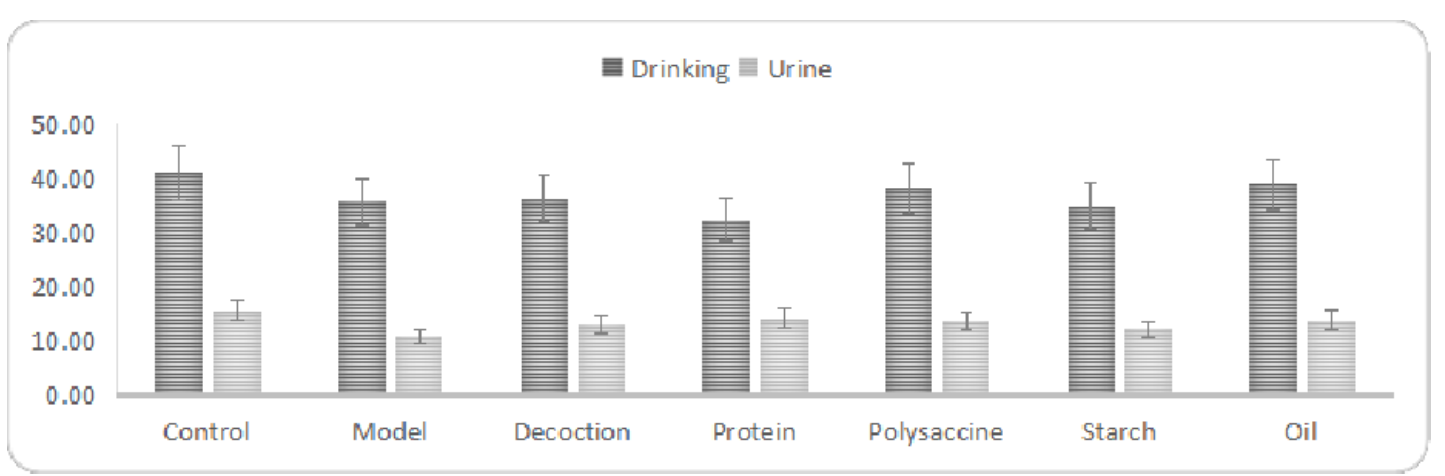

Figure 1. Covariance analyses of drinking volume and urine volume in each group.

The difference of the drinking volume was significant among groups $(P<0.05)$; the urine volume decreased significantly in the model group $(P<0.01)$; the urine volume increased significantly in the treatment groups $(P<0.05)$.

\section{Comparison of the water loading index in the treatment groups}

The water loading index of model group $(0.58)$ was higher than that of the control group $(P<0.01)$. The water loading index of the treatment groups decreased compared with that of the model group $(0.43,0.41,0.52,0.49,0.54)$ $(P<0.01)$. (Figure 2).

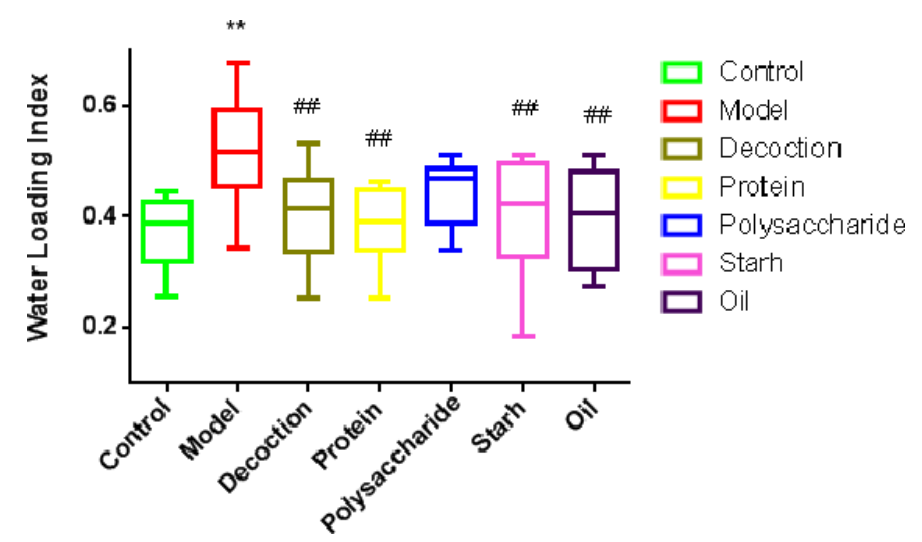

Figure 2: Comparison of water loading index in each group

The difference of the water lodading index was significant among groups $(P<0.05)$. Data was $\operatorname{mean} \pm \operatorname{SD}(\mathrm{n}=10$ rats per group). ${ }^{*} \mathrm{P}<0.05,{ }^{* *} \mathrm{P}<0.01$ vs control group. ${ }^{\#} \mathrm{P}<0.05,{ }^{\# \#} \mathrm{P}<0.01$ vs model group.

\section{PCA analysis of differential mRNA related to transport activity}

The model group and the control group could be clearly distinguished in the 3D chart. Bi-plot analysis showed: (1) There were three principal components (contribution rate of PC1, PC2, PC3=61.7\%, 15.3\%, 13\%); (2) The decoction group, the protein group and the polysaccharide group could improve the transport activity mRNA expression significantly, while the oil group had little effect (Figure 3). 
Han et al, Afr J Tradit Complement Altern Med., (2017) 14 (4): 239-246

https://doi.org/10.21010/ajtcam.v14i4.26

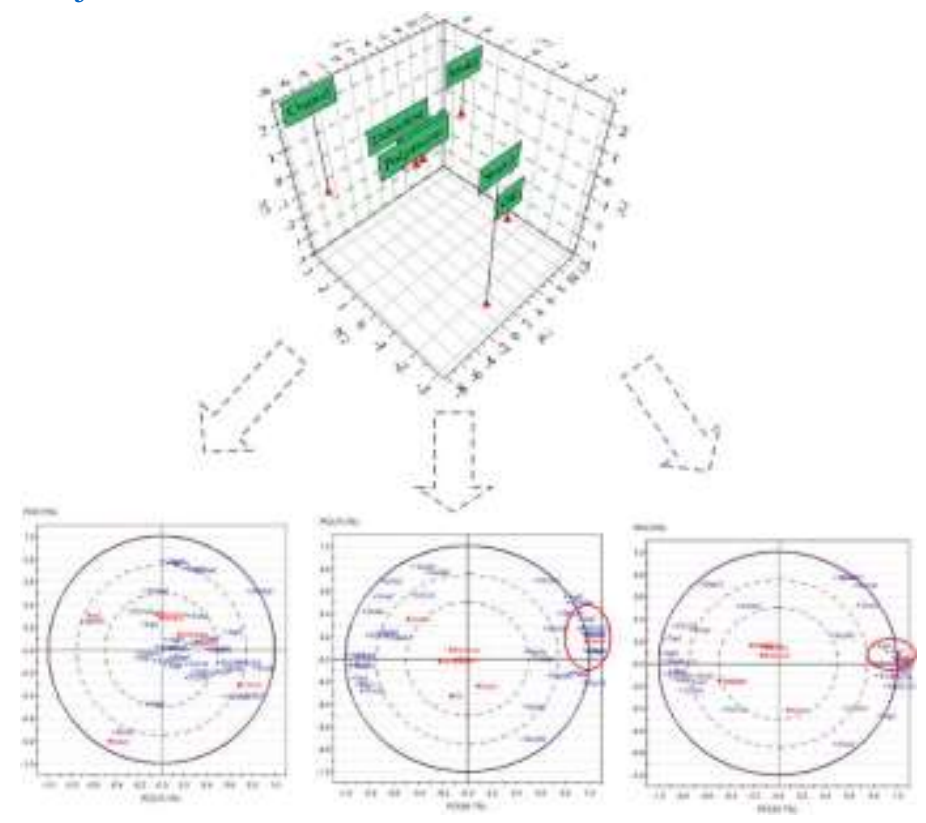

Figure 3: PCA analysis of mRNA microarrays.

The analysis of the normalized coefficient of PC1-3 showed that Fabp6, Slc51a, Slc51b, Slc11a2, Slc4a10 and AQP3 were the top six genes (Table 2, Table 3).

Table 2: Coefficient of different PC.

\begin{tabular}{|c|c|c|c|c|c|}
\hline PC1 & & PC2 & & PC3 & \\
\hline Variable & Coefficient & Variable & Coefficient & Variable & Coefficient \\
\hline Slc26a3 & 0.976 & Slc2a2 & 0.931 & Aqp8 & 0.892 \\
\hline Aqp7 & 0.938 & Slc51a & 0.829 & Sec1415 & 0.527 \\
\hline Slc51b & 0.938 & Fabp6 & 0.826 & Slc13a5 & 0.515 \\
\hline Aqp3 & 0.927 & Slc23a2 & 0.78 & Fabp6 & 0.481 \\
\hline RGD1565367 & 0.921 & Sec1415 & 0.63 & Slc2a2 & 0.324 \\
\hline Slc51a & 0.914 & Csn1s1 & 0.521 & Pmp2 & 0.286 \\
\hline Slc5a12 & 0.885 & Crabp1 & 0.479 & Csn1s1 & 0.276 \\
\hline Fabp6 & 0.87 & Pmp2 & 0.435 & Rbp7 & 0.266 \\
\hline Abcb1b & 0.867 & Slc4a1 & 0.414 & Slc51b & 0.247 \\
\hline Fabp4 & 0.856 & Aqp3 & 0.369 & Abcc6 & 0.237 \\
\hline Pmp2 & 0.719 & Abcc6 & 0.34 & Slc4a1 & 0.215 \\
\hline Csn1s1 & 0.708 & Aqp8 & 0.336 & Slc51a & 0.211 \\
\hline Slc13a5 & 0.559 & Tар2 & 0.318 & Slc23a2 & 0.174 \\
\hline Slco5a1 & 0.421 & Slc13a5 & 0.312 & Crabp1 & 0.17 \\
\hline Slc5a5 & 0.331 & Slc51b & 0.292 & Abcb1b & 0.063 \\
\hline Crabp1 & 0.311 & Aqp7 & 0.144 & Tap2 & 0.05 \\
\hline Rph3a & 0.311 & Rbp7 & 0.105 & Aqp9 & 0.035 \\
\hline Slc5a4 & 0.08 & Abcb1b & 0.093 & Slc11a2 & 0.016 \\
\hline Slc23a2 & 0.008 & Slc5a4 & -0.034 & Aqp3 & 0.01 \\
\hline Sec1415 & -0.001 & Slco5a1 & -0.038 & Slco5a1 & -0.005 \\
\hline Slc11a1 & -0.057 & Slc11a1 & -0.055 & Slc5a4 & -0.012 \\
\hline Slc5a1 & -0.231 & Fabp4 & -0.197 & Fabp4 & -0.12 \\
\hline Slc4a7 & -0.33 & Slc5a1 & -0.227 & Slc26a2 & -0.127 \\
\hline Slc2a2 & -0.494 & Aqp9 & -0.237 & Slc5a5 & -0.17 \\
\hline
\end{tabular}


Han et al, Afr J Tradit Complement Altern Med., (2017) 14 (4): 239-246

https://doi.org/10.21010/ajtcam.v14i4.26

\begin{tabular}{rrrrrr} 
Slc4a1 & -0.538 & Slc26a2 & -0.244 & Fabp12 & -0.208 \\
Slc26a2 & -0.542 & Fabp12 & -0.246 & Rph3a & -0.252 \\
Fabp12 & -0.58 & Slc5a12 & -0.249 & RGD1565367 & -0.264 \\
Aqp9 & -0.742 & Rph3a & -0.251 & Slc5a1 & -0.292 \\
Slc4a10 & -0.815 & Slc4a10 & -0.368 & Slc5a12 & -0.35 \\
Aqp8 & -0.832 & Slc4a7 & -0.37 & Slc26a3 & -0.353 \\
Abcc6 & -0.842 & RGD1565367 & -0.428 & Aqp7 & -0.36 \\
Rbp7 & -0.862 & Slc5a5 & -0.474 & Slc11a1 & -0.554 \\
Tap2 & -0.914 & Slc11a2 & -0.501 & Slc4a10 & -0.604 \\
Slc11a2 & -0.941 & Slc26a3 & -0.577 & Slc4a7 & -0.871 \\
\hline
\end{tabular}

Table 3: Normalized coefficient of each gene.

\begin{tabular}{cccc}
\hline Variable & Coefficient & Variable & Coefficient \\
\hline Fabp6 & 0.076456 & Slc13a5 & 0.030665 \\
Slc51a & 0.074885 & Abcc6 & 0.027684 \\
Slc51b & 0.062386 & Fabp12 & 0.02592 \\
Slc11a2 & 0.062317 & Slc26a2 & 0.021884 \\
Slc4a10 & .059034 & Slc4a7 & 0.020247 \\
Aqp3 & 0.057569 & Aqp8 & 0.017378 \\
Abcb1b & 0.045099 & Crabp1 & 0.011981 \\
Aqp7 & 0.044554 & Slco5a1 & 0.009314 \\
Csn1s1 & 0.044305 & Slc4a1 & 0.008408 \\
Pmp2 & 0.043495 & Slc5a1 & 0.006724 \\
Tap2 & 0.037581 & Sec1415 & 0.003918 \\
Aqp9 & Slc23a2 & 0.003133 \\
Fabp4 & 0.03479 & Rph3a & 0.002116 \\
Rbp7 & 0.033786 & Slc2a2 & 0.002099 \\
RGD1565367 & 0.033618 & Slc11a1 & 0.00194 \\
Slc26a3 & 0.031859 & Slc5a5 & 0.001744 \\
Slc5a12 & 0.0318 & Slc5a4 & 0.000263
\end{tabular}

mRNA name: Csn1sl:casein alpha sl;Abcblb:ATP-binding cassette, subfamily B (MDR/TAP), member 1B;Slc4a1:solute carrier family 4 (anion exchanger), member 1;Tap2:transporter 2, ATP-binding cassette, sub-family $B$ (MDR/TAP);Crabpl:cellular retinoic acid binding protein 1; Slc2a2:solute carrier family 2 (facilitated glucose transporter), member 2;Fabp6:fatty acid binding protein 6, ileal;Slc5al:solute carrier family 5 (sodium/glucose cotransporter), member 1; Slc1 la2:solute carrier family 11 (proton-coupled divalent metal ion transporter), member 2;Aqp7:aquaporin 7;Aqp8:aquaporin 8;Slc23a2:solute carrier family 23 (ascorbic acid transporter), member 2;Aqp9:aquaporin 9;Aqp3:aquaporin 3;Fabp4:fatty acid binding protein 4, adipocyte;Abcc6:ATP-binding cassette, subfamily $C$ (CFTR/MRP), member 6;Slc5a5:solute carrier family 5 (sodium/iodide cotransporter), member 5; Slc26a3:solute carrier family 26 (anion exchanger), member 3;Slc26a2:solute carrier family 26 (anion exchanger), member 2;Slc4a7:solute carrier family 4, sodium bicarbonate cotransporter, member 7;Rph3a:rabphilin 3A;Slc13a5:solute carrier family 13 (sodium-dependent citrate transporter), member 5;Sec14l5:SEC14-like 5 (S. cerevisiae); Slc5a4: solute carrier family 5 (glucose activated ion channel), member 4;Slc4al0:solute carrier family 4, sodium bicarbonate transporter, member 10;:Slc51b:solute carrier family 51, beta subunit; Slc51a:solute carrier family 51, alpha subunit;RGD1565367:similar to Solute carrier family 23, member 2 (Sodium-dependent vitamin C transporter 2); Slco5a1:solute carrier organic anion transporter family, member 5A1; Slc1 la1:solute carrier family 11 (proton-coupled divalent metal ion transporter), member 1; Slc5a12:solute carrier family 5 (sodium/monocarboxylate 
Han et al, Afr J Tradit Complement Altern Med., (2017) 14 (4): 239-246

https://doi.org/10.21010/ajtcam.v14i4.26

cotransporter), member 12;Rbp7:retinol binding protein 7, cellular;Fabp12:fatty acid binding protein 12;Pmp2:peripheral myelin protein 2.

\section{qRT-PCR Detection}

qRT-PCR was used to validate the accuracy of mRNA microarrays. AQP3 and AQP8 were selected randomly. Spearman Rho correlation analysis showed that there was a significant correlation between mRNA microarrays and qRTPCR $(r=0.956$, Sig = 0.000) (Figure 4).

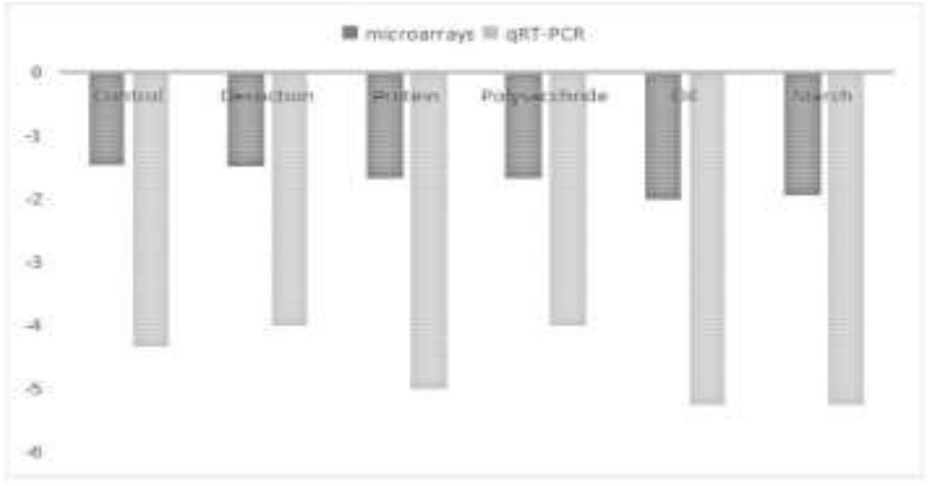

A

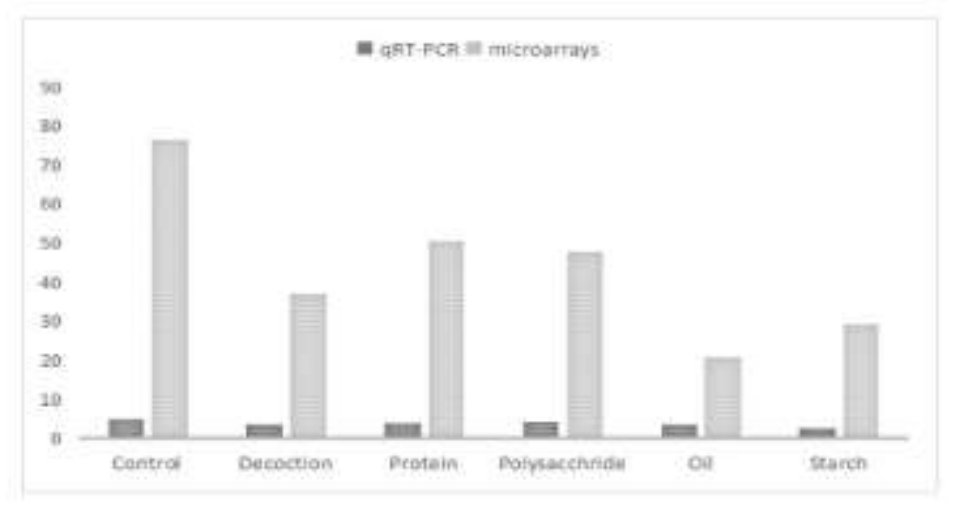

B

Figure 4: Correlation between qRT-PCR and mRNA microarrays (A:AQP3, B:AOP8)

\section{Discussion}

Spleen deficiency and wet dampness (SDWD) ZHENG is one of the major syndromes of TCM, of which excessive fatigue and high fatty diet are main causes (Lim et al., 2015). SDWD can lead to the imbalance of gastrointestinal functions, edema and diarrhea, etc. (Gao et al., 2014, Han et al., 2015), which is frequently treated with fortifying the spleen and inhibiting the dampness clinically (Dai et al., 2016).

Coix seed is frequently used to treat diseases related to SDWD in TCM (Huang et al., 2014, Zhao et al., 2014). However, the effective components of fortifying the spleen and inhibiting the dampness are not clear. In previous study four components have been extracted from Coix seed.

The results show the water load index in all treatment groups decreases and the urine volume increases, which indicates that the active components of Coix seed can regulate the water transport of SDWD rats, among which the components of proteins and polysaccharides play the major role.

Microarray-based gene expression analysis is widely used in the study of TCM. It has already been applied in the study of clinical diseases (Khraiwesh et al., 2015). It detects the whole genome of the objects, which may provide possible therapeutic effect and clarify the mechanism. A lot of researches had been done in ZHENG fields with mRNA microarrays technology (Sun et al., 2016). Fang et al. found the relationship between neuroendocrine-immune and different ZHENG in tumor-bearing mice (Fang et al., 2010). mRNA microarrays is a good choice for us to explore the nature of SDWD.

The results show that SDWD model rats have higher expression of Abcb1b, Fabp6, Aqp7, Aqp3, Fabp4, Slc26a3, Slc13a5, Slc51b, Slc51a gene and lower expression of Slc4a1, Slc2a2, Slc5a1, Slc23a2, Slc26a2, Slc4a7, Slc5a4, Slc11a1 gene, which is consistent with Ying's research results (Ying et al., 2016). The genes are closely 244 
related to water transport, which is the mechanism of SDWD water accumulation. WK Adams also believes that longterm diet disorders cause the body's water and salt metabolism deficiency (Adams et al., 2015). Our previous studies find that the expression of Slc and AQP abnormally in SDWD rats and the expression of Apoa5 and Apoe improves (HAN et al., 2016, Han et al., 2016), which indicates that there is abnormal water transport in model rats.

After treatment with the components of Coix seed, the rat models show improvement of SDWD ZHENG. Principal component analysis shows that the loading of differential mRNA of the protein group and the polysaccharide group was closer to that of the decoction group, and the loading of the oil group was closer to the model group. It suggests that the components of proteins and polysaccharides play a more significant role in normalizing the mRNA expression of SDWD model rats. The most significant gene is Fabp6, Slc51a, Slc51b, Slc11a2, Slc4a10 and AQP3 respectively, which indicates that the components of proteins and polysaccharides play an important role in regulating water transport via the Slc gene and AQP gene family.

It is note-worthy that the result of mRNA microarrays are not entirely consistent with the results of water loading index, which shows that the components of oils and starches may have other ways in fortifying the spleen and inhibiting the dampness, which is worthy of further study.

\section{Conclusion}

The results from the present study demonstrate that components of Coix seed have excellent effect in treating SDWD by fortifying the spleen and inhibiting the dampness. The components of proteins and polysaccharides play an important role in regulating water transport, the components of oils and starches may play a part in other way.

\section{Acknowledgments}

This work was supported by the National Key Basic Research Program of China (973, NO.2013CB531803); National Natural Science Foundation of China (NO.81673852); Shandong province administration of TCM (NO.2015008, NO.2015-016).

\section{Ethics statement}

The study was approved by the experimental animal center of Shandong University of Traditional Chinese Medicine. The animals were fed in individually ventilated cages (IVCs), the housing facility conformed to the national standard "Laboratory Animals-Requirements for Environment and Housing Facilities" (GB 14925-2001).

\section{References}

1. Adams, W.K., J.L. Sussman, S. Kaur, M. D'Souza A, T.J. Kieffer, and C.A. Winstanley. (2015). Long-term, calorie-restricted intake of a high-fat diet in rats reduces impulse control and ventral striatal D2 receptor signalling - two markers of addiction vulnerability. Eur J Neurosci.,42: 3095-3104.

2. Cui, N., W. Zhao, B. Han, X. Ji, J. Gao, X. Han, and S. Wang. (2016). Evaluation of rat model of spleen deficiency with water and dampness based on factor analysis. Journal of Basic Chinese Medicine.,22: 35-37.

3. Cui, Y., R. Rong, Y. YANG, and L. GONG. (2015). Optimization of ultrasound-assisted acid extraction of polydaccharide from Coix seed. Chinese Archives of Traditional Chinese Medicine.,33: 2163-2146.

4. $\quad$ Cui, Y., X.-m. Wang, Y. Yang, L.-1. Gong, H.-q. Jiang, and B.-b. Han. (2014). Research on fusion-fingerprint of Coicis Semen oil. Chinese Traditional and Herbal Drugs.,45: 1698-1701.

5. Dai, B., Q. Wu, C. Zeng, J. Zhang, L. Cao, Z. Xiao, and M. Yang. (2016). The effect of Liuwei Dihuang decoction on PI3K/Akt signaling pathway in liver of type 2 diabetes mellitus (T2DM) rats with insulin resistance. $\mathbf{J}$ Ethnopharmacol.,4: 1872-7573.

6. Fang, Z., Z. Pan, W. Lu, X. Liu, D. Guan, C. Liang, and B. Gao. (2010). Genes transcription characteristic in neuroendocrine-immune-network about typical syndrome in tumor-bearing mice. Chinese Journal of Basic Medicine in Traditional Chinese Medicine.,16: 33-36.

7. GAO, J., B.-b. HAN, X. HAN, N. Cui, W.-x. Zhao, and S.-j. Wang. (2014). Experimental Study on General Status of Rat Models with Spleen Deficiency. Information on Traditional Chinese Medicine.,86-87.

8. Gao, J., B. Han, and X. Han. (2014). Changes in Gastrointestinal Functions of Rat with Dampness Stagnancy due to Spleen Deficiency induced by Low- protein and Low- choline Diet Plus Swimming. Chinese Archives of Traditional Chinese Medicine.,32: 2667-2668.

9. HAN, X.-c., X.-m. JI, Y.-f. WANG, Y.-n. ZHANG, Q. CHEN, Z.-x. PANG, H.-r. WANG, J. YUE, Y. REN, and S.-j. WANG. (2016). Data mining on genic change of intercellular space component of gene chip on rats with syndrome of fluid-dampness due to spleen deficiency intervened by different components in coix seed. China Journal of Traditional Chinese Medicine and Pharmacy., 31: 1955-1958. 
10. Han, X., B.-b. Han, J. Gao, N. Cui, W.-x. Zhao, and L.-q. Liu. (2015). Effect on Liver Function of Rats with High-fat and Low-protein Diet. Information on Traditional Chinese Medicine.,0:60-62.

11. Han, X., X. Ji, Y. Zhang, Y. Wang, Y. Yin, And Wang, S. (2016). Effect of different components of Coix Seed on related genes in jejunal basolateral membrane of rats with syndrome of spleen deficiency and water dampness. Journal of Beijing University of Traditional Chinese Medicine.,39: 466-469.

12. Huang, X., J. Qin, and S. Lu. (2014). Kanglaite stimulates anticancer immune responses and inhibits HepG2 cell transplantationinduced tumor growth. Mol Med Rep.,10: 2153-9.

13. Jie, G., H. Xu, H. Bing-bing, C. Ning, Z. Wen-xiao, and W. Shi-jun. (2015). Changes in Immune Functions of Rat Models with Dampness Stagnancy due to Spleen Deficiency Induced by Compound Molding Factors. Information on Traditional Chinese Medicine.,0: 40-42.

14. Khraiwesh, B., E. Qudeimat, M. Thimma, A. Chaiboonchoe, K. Jijakli, A. Alzahmi, M. Arnoux, and K. SalehiAshtiani. (2015). Genome-wide expression analysis offers new insights into the origin and evolution of Physcomitrella patens stress response. Sci Rep.,5: 17434.

15. Lim, D.H., Y.G. Kim, S.H. Bae, S. Ahn, S. Hong, C.K. Lee, and B. Yoo. (2015). Factors related to outcomes in lupus-related protein-losing enteropathy. Korean J Intern Med.,30: 906-12.

16. Liu, J., T. Jiang, and J. Li. (2016). Application progress of proteomics for TCM syndrome research. China Journal of Traditional Chinese Medicine and Pharmacy.,31: 196-199.

17. Liu, X., Z. Fang, Z. Pan, W. Lu, Z. Wu, C. Liang, and Y. Zhang. (2014). Pituitary transcriptome profile of liver cancer mice with different syndromes reveals the relevance of pituitary to the cancer and syndromes. $J$ Tradit Chin Med.,34: 691-698.

18. Pedersen, R.S., H. Bentzen, J.N. Bech, O. Nyvad, and E.B. Pedersen. (2003). Urinary aquaporin-2 in healthy humans and patients with liver cirrhosis and chronic heart failure during baseline conditions and after acute water load. Kidney Int.,63: 1417-25.

19. Saito, T., M. Higashiyama, T. Nakamura, I. Kusaka, S. Nagasaka, T. Saito, and S. Ishikawa. (2001). Urinary excretion of the aquaporin-2 water channel exaggerated in pathological states of impaired water excretion. Clin Endocrinol (Oxf),,55: 217-21.

20. Sun, A., Z. Yuan, S. Xia, and Y. Zhang. (2016). Current status and prospect of system blology research on TCM syndrom. China Journal of Traditional Chinese Medicine and Pharmacy.,31: 200-205.

21. Wu, H., L.L. Gong, Y. Yang, R. Rong, and J.Q. Ju. (2015). Study on Optimum Extraction Process of Water Soluble Protein from Coicis Semen. Zhong Yao Cai.,38: 376-80.

22. Yang, Z.A.-O., L.H. Chen, M. Hong, Y.A.-O.X. Chen, X.R. Yang, S.M. Tang, Q.F. Yuan, Z.Y. He, and W.W. Chen. (2016). Serum MicroRNA Profiling and Bioinformatics of Patients with Spleen-Deficiency Syndrome. Evid Based Complement Alternat Med.,20: 1741-427X (Print).

23. Ying, W., J. Lianqun, S. Nan, C. Yuan, C. Huimin, L. Jingjing, and Y. Guanlin. (2016). Expression Changes of mRNA on Lipoprotein and Cholesterol Metabolism Signaling Pathways in Liver of Spleen Deficiency and Phlegm Turbidity Syndrome Rats by PCR Array Technology. Liaoning Journal of Traditional Chinese Medicine.,43: 145-148.

24. You-ming, L., L. Yi-ming, and L. Mei-juan. (2011). Effect of the digestive system disease treated by adlay. J Tradit Chinese Medicine.,52: 1334-1335.

25. Zhao, M., D. Zhu, D. Sun-Waterhouse, G. Su, L. Lin, X. Wang, and Y. Dong. (2014). In vitro and in vivo studies on adlay-derived seed extracts: phenolic profiles, antioxidant activities, serum uric acid suppression, and xanthine oxidase inhibitory effects. J Agric Food Chem.,62: 7771-8. 